\title{
POSTPROCESSING AND VISUALIZATION TECHNIQUES FOR CONVECTION-ALLOWING ENSEMBLES
}

\author{
Brett Roberts, Israel L. Jirak, Adam J. Clark, Steven J. Weiss, and John S. Kain
}

To supplement the increasing operational presence of convection-allowing ensemble forecast systems, approaches for displaying their most useful output are demonstrated and discussed.

N early three decades ago, Lilly (1990) suggested the time had arrived for numerical weather prediction (NWP) to evolve toward explicit forecasts of convective storms. Over the years that followed, the research community made tremendous strides in laying the groundwork for operational

AFFILIATIONS: ROBERTS-Cooperative Institute for Mesoscale Meteorological Studies, and NOAA/NWS/Storm Prediction Center, and NOAA/OAR/National Severe Storms Laboratory, Norman, Oklahoma; JIRAK AND WEISS-NOAA/NWS/Storm Prediction Center, Norman, Oklahoma; CLARK-NOAA/OAR/ National Severe Storms Laboratory, and School of Meteorology, University of Oklahoma, Norman, Oklahoma; KAIN*_NOAA/ OAR/National Severe Storms Laboratory, Norman, Oklahoma * CURRENT AFFILIATION: NOAA/NCEP/Environmental Modeling Center, College Park, Maryland CORRESPONDING AUTHOR: Brett Roberts, brett.roberts@noaa.gov

The abstract for this article can be found in this issue, following the table of contents.

DOI:I0.II75/BAMS-D-I8-004I.I

In final form 14 November 2018

(C)2019 American Meteorological Society

For information regarding reuse of this content and general copyright information, consult the AMS Copyright Policy. convection-allowing models (CAMs) and, in collaboration with operational forecasters, demonstrating their predictive value relative to coarser-grid models that incorporate parameterized convection (e.g., Droegemeier 1997; Weisman et al. 1997; Xue et al. 2003; Done et al. 2004; Kain et al. 2006; Liu et al. 2006; Clark et al. 2007; Weisman et al. 2008). As high performance computing (HPC) resources increased and allowed the NWP community to run the first realtime deterministic CAMs in the early-to-mid 2000s, modelers and forecasters faced an abundance of new model data, but exploiting these data for operational forecasting applications presented new challenges. Traditional NWP outputs like thermodynamic and kinematic variables and quantitative precipitation forecasts (QPFs) can provide much useful information from a coarser-grid NWP model incorporating parameterized convection, but they fail to convey much of the value-added information available from a CAM, necessitating new or modified diagnostic parameters. For example, computing simulated reflectivity (Koch et al. 2005) using an observation operator appropriate for the active microphysics scheme (e.g., Ferrier 1994; Jung et al. 2008) can help to characterize simulated convective structures in a display format familiar to forecasters and directly comparable to 
observed radar imagery. The vertical velocity field in a CAM can be used to assess updraft and downdraft strength explicitly within convective cells, in addition to the much broader and weaker perturbations tied to quasigeostrophic processes. Updraft helicity (UH; Kain et al. 2008) is an important diagnostic new to the CAM era that identifies rotating updrafts (e.g., supercells) in simulated convection with remarkable efficacy. $\mathrm{UH}$ in CAMs was initially examined in test bed environments like the NOAA Hazardous Weather Testbed (e.g., Kain et al. 2008; Clark et al. 2012a; Gallo et al. 2017), and is now commonly used in convective forecasting operationally (e.g., Guyer and Jirak 2014). Given the correspondence between supercells and observed severe weather, postprocessing techniques that use $\mathrm{UH}$ as their sole input have even demonstrated considerable value in highlighting the threat for severe convective hazards (e.g., Sobash et al. 2011, 2016b). Although the application of UH to tornado forecasting has received the most attention among these hazards (e.g., Clark et al. 2012b; Gallo et al. 2016), it has also been applied successfully to operational guidance for hail and severe wind gusts (e.g., Jirak et al. 2014).

The development of new diagnostic fields appropriate for CAM-simulated convection advanced insights available from finer-scale models, but also presented yet another concurrent challenge: How should the short time scales inherent to these diagnostics be represented usefully within our existing visualization paradigms for gridded NWP data, which typically present fields to users at time intervals of hours? While forecasters can sample instantaneous snapshots of traditional NWP fields (e.g., 500-mb geopotential height and others commonly used to assess synoptic-scale features) every $1-6 \mathrm{~h}$ during the model forecast and capture most information relevant to their conceptual understanding of the forecast scenario, this is not true for diagnostic CAM fields tied to explicitly simulated convection, as these often evolve in important ways on time scales of minutes. A simulated thunderstorm may in some instances develop, mature, and dissipate within a 1-h period, such that sampling the model state at hourly intervals would give little useful information about its structure or evolution, and might even provide misleading information to a forecaster. However, displaying the model state at subhourly intervals would entail very large data dissemination bandwidth requirements, and such an approach would also necessitate enhancing existing operational workstation capabilities and visualization systems. To communicate data from the relevant time scales succinctly without overwhelming operational infrastructure, Kain et al. (2010) recommended that modelers track time-maximum (and/or time-minimum) values of fields like $\mathrm{UH}$ and vertical velocity during model integration at each grid point and time step over time bins useful to forecasters. This approach has since been widely embraced in CAM NWP, and presently, hourly-maximum fields ${ }^{1}$ (HMFs) for CAM diagnostics are often used as inputs for postprocessing tasks.

The innovations described thus far were forged during an era when real-time CAMs were in their early stages of development and took the form of individual deterministic runs, often experimental in nature. As deterministic CAMs have matured and been implemented in NWS operations, real-time CAM ensemble systems have also become a reality. Starting in 2007, the first experimental real-time CAM ensemble was tested in the NOAA Hazardous Weather Testbed (HWT) Spring Forecasting Experiment (SFE; Xue et al. 2007). Accordingly, it is the intersection of CAMspecific and ensemble-specific visualization and interpretation paradigms which now constitutes a new frontier for CAM NWP postprocessing. The purpose of this article is to update the broader meteorological community on the current state of CAM ensemble postprocessing techniques, products, and visualizations in preparation for their increased operational presence in the coming years. In the next section, we present a brief overview of the High Resolution Ensemble Forecast system, a CAM ensemble whose recent operational implementation has in part motivated this article. In the subsequent two sections, we outline some of the current solutions for condensing and presenting useful CAM ensemble output in operational NWP postprocessing. In the final section, we summarize the current state of CAM ensemble postprocessing and preview some potential future directions.

\section{NCEP'S HIGH RESOLUTION ENSEMBLE FORECAST SYSTEM. The High Resolution} Ensemble Forecast system version 2 (HREFv2) was implemented operationally at the National Centers for Environmental Prediction (NCEP) on 1 November 2017. The HREFv2 is NCEP's first operational CAM ensemble and in many respects mirrors a proof-ofconcept originally demonstrated by the Storm Scale Ensemble of Opportunity (SSEO; Jirak et al. 2012), a multimodel CAM ensemble processed experimen-

\footnotetext{
${ }^{1}$ At a grid point, the HMF of a variable (e.g., UH) is simply the maximum value the variable attained at any model time step over the previous hour at the point.
} 
tally at the Storm Prediction Center (SPC) and widely utilized from 2011 to 2017. The original motivation for the SSEO was to summarize and efficiently aggregate the output from a small number of deterministic regional CAMs available at the time of its inception. The SSEO formally consisted of seven members, with five members run operationally at its inception, and two other members run experimentally that were subject to periodic delays and outages. A key feature of the SSEO membership was diversity across several dimensions: model cores, parameterization schemes, initialization times (i.e., time-lagged members were used), and initial and lateral boundary conditions (Jirak et al. 2012). The HREFv2 employs a similar design for its membership, but all of its members are run operationally at NCEP on an optimized schedule that is much timelier than the SSEO for use by operational forecasters.

Table 1 summarizes the configurations of each HREFv2 ensemble member that is postprocessed at the SPC in mid-2018. The HREFv2 consists of eight members with four deterministic CAM configurations employed, and each configuration is represented in two members because of the time-lagging approach. Two dynamical cores are represented: the Advanced Research version of the Weather Research and Forecasting Model (WRF-ARW; Skamarock et al. 2008) and the Nonhydrostatic Multiscale Model on the B Grid (NMMB; Janjić and Gall 2012). Diversity also exists with respect to microphysics and planetary boundary layer (PBL) parameterization schemes, along with initial and lateral boundary conditions, which are provided by coarser-grid NWP models [i.e., Rapid Refresh (RAP) and North American Mesoscale Forecast System (NAM)]. Subjective and objective evaluation metrics collected during the 2017 and 2018 HWT SFEs indicated that the HREFv2 outperformed several more formally designed ${ }^{2}$ experimental CAM ensembles in depicting features relevant to forecasting severe convection, such as the correspondence between ensemble UH maxima and observed storm reports (Jirak et al. 2018).

Coinciding with the HREFv2 implementation, the SPC HREF Ensemble Viewer (www.spc.noaa.gov lexper/href) was launched on 1 November 2017. This web-based tool for displaying HREF data is available on the public SPC website and offers many of the products discussed in this article on a real-time basis, with images for two daily HREFv2 cycles (0000 and 1200 UTC) appearing a few hours after their respective initialization times. Most of the figures hereafter are sourced from the SPC HREF Ensemble Viewer.

\footnotetext{
${ }^{2}$ Other CAM ensembles evaluated in the SFE generally employ a unified model configuration (model core, grid, parameterization schemes, etc.) among their members; ensemble spread is achieved primarily through formal techniques that apply disparate perturbations to the initial conditions of each member. By contrast, HREFv2 members use disparate model cores, parameterization schemes, and parent models from which their initial and lateral boundary conditions are inherited. These configuration differences yield ensemble spread, obviating the need for formal perturbation strategies.
}

TABLE I. Member configurations for the HREFv2, as postprocessed at the SPC in mid-20I8. HRW and NAM refer to High Resolution Window and North American Mesoscale Forecast System runs, respectively. In the "initial/boundary conditions" column, the parent NWP system providing the background for each member is specified; "-6 h" indicates that the parent model was initialized $6 \mathrm{~h}$ prior to the nested CAM, which inherits its background. The WRF single-moment 6-class microphysics scheme (WSM6) is described in Hong and Lim (2006); the Ferrier-Aligo microphysics scheme is described in Aligo et al. (20I8); the Mellor-Yamada-Janjić (MYJ) PBL scheme is described in Janjić (1994); and the Yonsei University (YSU) PBL scheme is described in Hong et al. (2006).

\begin{tabular}{|lccccc|}
\hline Member & Model core & Time-lagged? & $\begin{array}{c}\text { Initial/boundary } \\
\text { conditions }\end{array}$ & Microphysics & PBL \\
\hline HRW NSSL & WRF-ARW & $\mathrm{N}$ & NAM/NAM $-6 \mathrm{~h}$ & WSM6 & MYJ \\
\hline HRW ARW & WRF-ARW & $\mathrm{N}$ & RAP/GFS $-6 \mathrm{~h}$ & WSM6 & YSU \\
\hline HRW NMMB & NMMB & $\mathrm{N}$ & RAP/GFS $-6 \mathrm{~h}$ & Ferrier-Aligo & MYJ \\
\hline NAM Nest & NMMB & $\mathrm{N}$ & NAM/NAM & Ferrier-Aligo & MYJ \\
\hline HRW NSSL $-12 \mathrm{~h}$ & WRF-ARW & $\mathrm{Y}(-12 \mathrm{~h})$ & NAM/NAM $-6 \mathrm{~h}$ & WSM6 & MYJ \\
\hline HRW ARW $-12 \mathrm{~h}$ & WRF-ARW & $\mathrm{Y}(-12 \mathrm{~h})$ & RAP/GFS $-6 \mathrm{~h}$ & WSM6 & YSU \\
\hline HRW NMMB $-12 \mathrm{~h}$ & NMMB & $\mathrm{Y}(-12 \mathrm{~h})$ & RAP/GFS $-6 \mathrm{~h}$ & Ferrier-Aligo & MYJ \\
\hline NAM Nest $-12 \mathrm{~h}$ & NMMB & $\mathrm{Y}(-12 \mathrm{~h})$ & NAM/NAM & Ferrier-Aligo & MYJ \\
\hline
\end{tabular}


SPATIAL NEIGHBORHOOD APPROACH FOR HMF ENSEMBLE SUMMARIES. As HPC

capacities have increased and NWP grid spacing has decreased over time, and as CAMs capable of resolving some convective structures have emerged, limits to the predictability of individual convective storms have become important to consider in a practical sense. For example, it is unlikely that CAMs will routinely represent a predicted feature in precisely the same grid cell and at the exact time where the feature actually occurs in observations. Furthermore, even if a simulated single-cell thunderstorm is displaced horizontally from the observed storm by, say, three or four grid points in a CAM with 3-km grid spacing, the forecast would still likely be considered highly useful for many forecast applications. This has encouraged the development of spatial verification metrics with the property that "close misses" in space are given due credit, in accordance with subjective impressions regarding forecast value. One prevalent class of these metrics can be called the spatial "neighborhood" approach, wherein a neighborhood of grid points surrounding the point being verified is considered (Ebert 2009; Ahijevych et al. 2009).

In the context of NWP verification, neighborhood techniques can be applied to both deterministic and ensemble forecasts. Neighborhood approaches can also be leveraged for NWP postprocessing, where their application is most appropriate in the context of ensemble systems (i.e., considering the distribution of ensemble member solutions within the neighborhood). In this article, we will focus on neighborhood postprocessing applications. As explained at length by Schwartz and Sobash (2017, hereafter SS17), there are numerous specific implementations within this class of techniques, and they differ in some important ways. The neighborhood maximum ensemble probability (NMEP) defined in SS17 is a computational method intended to address prediction of localized high-impact events, making it ideally suited for summarizing HMFs in a CAM ensemble. Crucially, for a gridded field, the NMEP represents the probability that a selected parameter threshold will be exceeded anywhere within the neighborhood. This is in contrast to what SS17 called a neighborhood ensemble probability (NEP), which represents the fractional coverage of threshold exceedance within the neighborhood. The
NEP is a smoothing approach and effectively treats each grid point in the neighborhood as an additional ensemble member, such that expanding the neighborhood size results in more aggressive spatial smoothing and decreased forecast sharpness. Both NEPs and NMEPs help to fill out probability density functions (PDFs) that, for ensembles of limited membership size, would otherwise be highly discontinuous in the absence of these postprocessing techniques.

Although a fixed neighborhood size is traditionally employed for most neighborhood calculations, Blake et al. (2018, hereafter B18) have proposed applying the Ensemble Agreement Scale (EAS) technique first developed by Dey et al. (2016) to increase (decrease) the neighborhood length scale locally with decreasing (increasing) local agreement among ensemble members. In the case of HREF quantitative precipitation forecasts over 6-h periods, B18 demonstrated that using an adaptive neighborhood length scale for NEPs at 0.5 -in. and 1-in. exceedance thresholds can produce probabilistic forecasts that are more reliable than either grid point probabilities or fixed-scale NEPs. For NMEPs, however, a fixed neighborhood size is generally required, since the neighborhood length scale defines the search radius and therefore the fundamental physical meaning of the field (as opposed to only the smoothing radius for NEPs).

The NMEP approach used for the SPC HREF Ensemble Viewer computes all probabilities on the $3-\mathrm{km}$ HREF grid, ${ }^{3}$ so there is no upscaling step involving a coarser grid (e.g., Sobash et al. 2016b). Relative to upscaling approaches, this procedure increases computational cost, but retains higher spatial detail in the output field. For HMFs relevant to severe convective hazards (e.g., UH, updraft speed, and $10-\mathrm{m}$ wind speed), a square ${ }^{4}$ neighborhood of $80 \mathrm{~km} \times 80 \mathrm{~km}$ centered on each grid point is used to calculate the NMEP field with respect to selected HMF exceedance threshold(s), meaning the search "radius" is $40 \mathrm{~km}$ (although using a square rather than circular neighborhood). This choice of neighborhood size is intentionally matched to the spatial scale of the SPC's Convective Outlook products, which forecast probabilities of severe hazard occurrence within $40 \mathrm{~km}$ of a point.

Figure 1 illustrates the transformation of an ensemble HMF field to the NMEP fields plotted on the

\footnotetext{
${ }^{3}$ The native model grid differs slightly among the HREF members (e.g., WRF-ARW vs NMMB members), but all members are interpolated to a common 3-km grid by NCEP before data dissemination and postprocessing.

${ }^{4}$ A square neighborhood is used in lieu of a circular neighborhood for computational efficiency. When performing verification of NMEP forecasts, it is important to ensure the neighborhood scale applied to the observational dataset is matched correctly to that of the NMEP field.
} 
(a) Member 1, 24-h max UH $\left(\mathrm{m}^{2} \mathrm{~s}^{-2}\right)$

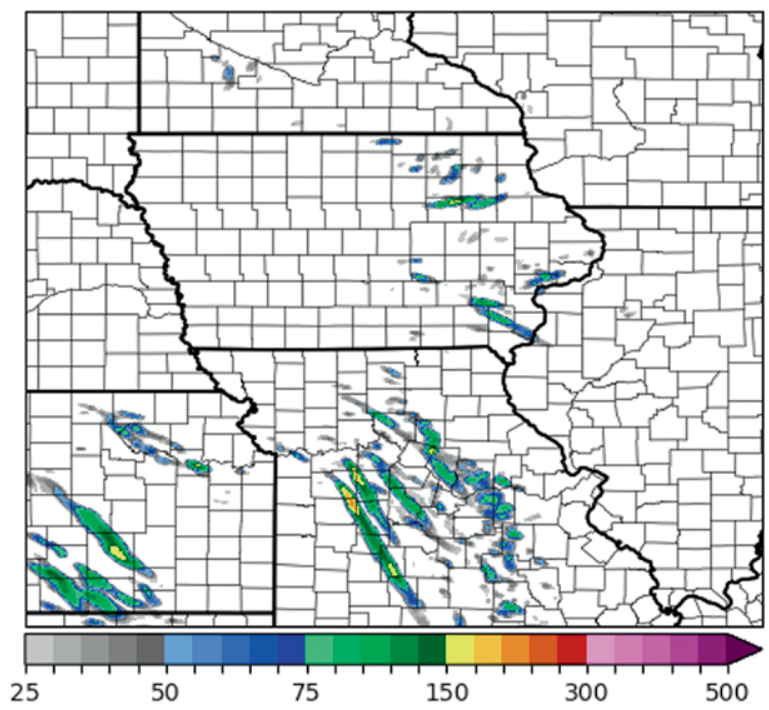

(c) Grid point NMEP (\%), UH $>75 \mathrm{~m}^{2} \mathrm{~s}^{-2}$

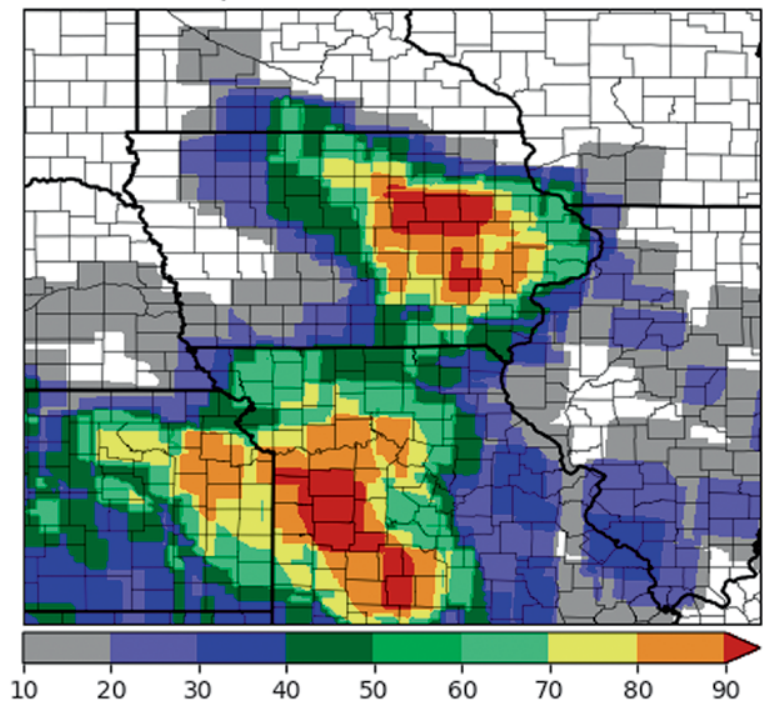

(b) Member 1, binary field ( $\left.\mathrm{UH}>75 \mathrm{~m}^{2} \mathrm{~s}^{-2}, \mathrm{r}=40 \mathrm{~km}\right)$

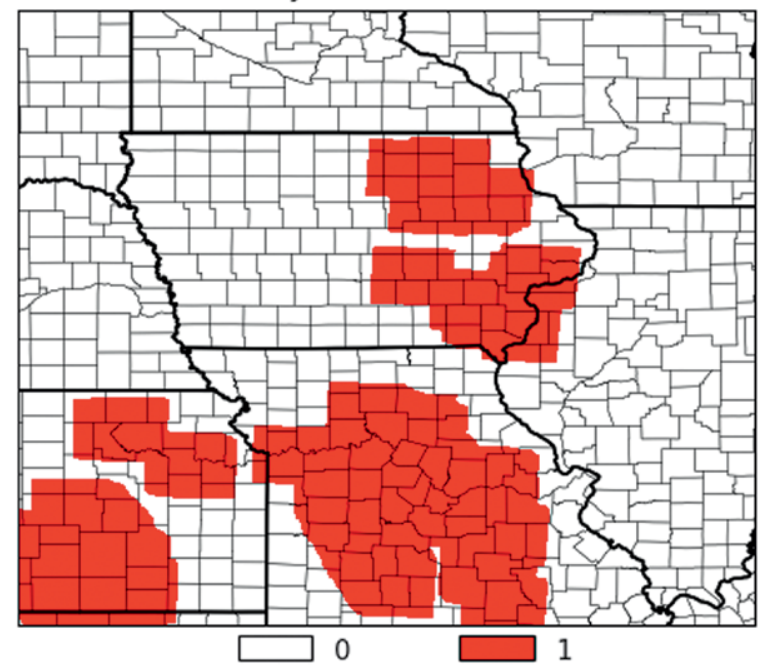

(d) Smoothed NMEP (\%), UH $>75 \mathrm{~m}^{2} \mathrm{~s}^{-2}$

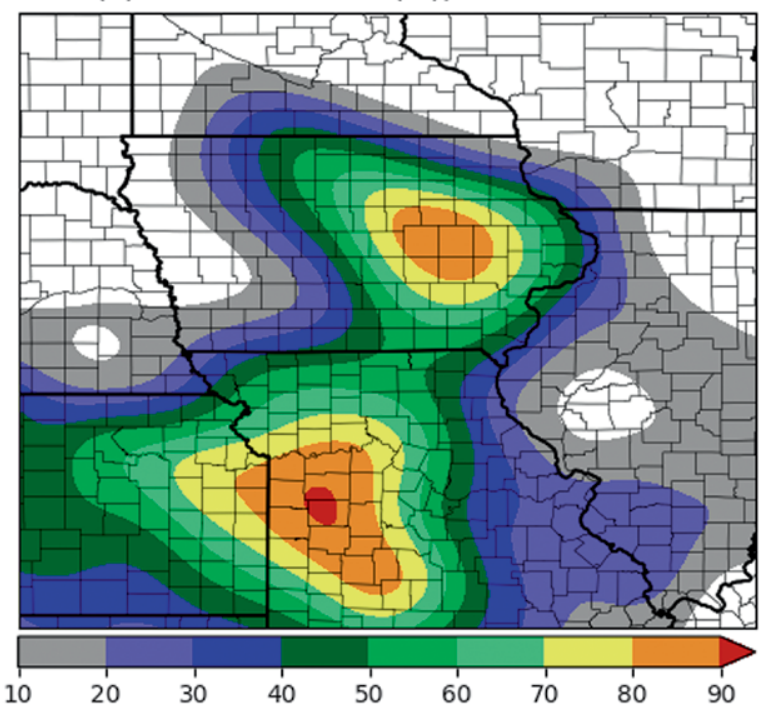

FIG. I. Step-by-step illustration of the procedure for computing the NMEP field for an HMF, as processed for the SPC HREF Ensemble Viewer. (a) The 24-h maximum UH (color fill; $\mathrm{m}^{2} \mathrm{~s}^{-2}$ ) for member I of a CAM ensemble forecast. (b) For member I, the binary field generated by setting values to $I$ at any grid point where a value of $\mathrm{UH}>75 \mathrm{~m}^{2} \mathrm{~s}^{-2}$ occurs within an $80 \mathrm{~km} \times 80 \mathrm{~km}$ neighborhood centered on the point, and 0 elsewhere. (c) The "grid point" NMEP field, which is equivalent to the average of the binary fields generated for all ensemble members. (d) The smoothed NMEP field using a Gaussian smoother with $\sigma=40 \mathrm{~km}$; this is equivalent to the NMEP field plotted on the SPC HREF Ensemble Viewer.

SPC HREF Ensemble Viewer. For each member, the HMF field (Fig. 1a) is first transformed into a binary field that is 1 where the HMF threshold is exceeded at any point in the local neighborhood, and 0 elsewhere (Fig. 1b). Next, the binary fields for all ensemble members are averaged to produce the "grid point" NMEP (Fig. 1c; plotted as a percentage). Finally, a Gaussian smoother [i.e., Eq. (6) in SS17] is applied with $\sigma=40 \mathrm{~km}$. This smoothed field is what appears on the final plots (Fig. 1d). As noted in SS17, smoothing the NMEP field in this way fills out the PDFs more realistically and yields plots without distracting discontinuity artifacts, properties that are particularly beneficial for smaller ensembles like the HREFv2.

The NMEP can be computed with respect to any exceedance threshold on any gridded field. For HMFs, it is most commonly computed with respect to the temporal maximum of the HMF over a single- or multi-hour period. Although presently it is common practice for CAMs to output HMFs at sequential 
hourly intervals in the data disseminated to downstream users, these HMFs can be considered across any combination of hourly bins during postprocessing to produce useful visualizations and products that are directly relevant to forecaster needs. For example, in support of operational convective forecasting activities at the SPC, there are two standard temporal scales for which postprocessed HMF-based products are generated. The first temporal scale is the 24-h maximum HMF over the convective day (1200-1200 UTC) corresponding to Convective Outlook products, a window that cleanly captures the diurnal maximum in convective activity across the United States. The second temporal scale is a rolling 4-h maximum HMF window computed hourly throughout the convective day (1200-1600 UTC, 1300-1700 UTC, etc.). The purpose of 4-h maximum HMF products is to highlight the peak likelihood of severe weather occurrence for any given point on a time scale appropriate for the phenomena in question. Krocak and Brooks (2017) found that, at a given point in the United States on a given day, more than 95\% of severe weather reports within $40 \mathrm{~km}$ of the point occur within a 4 -h period. Thus, it is appropriate at each point to identify the 4 -h period within the convective day when severe weather is most likely to occur. This philosophy underpins experimental outlook products currently in development at the SPC, and 4-h maximum HMF products from CAM ensembles will be essential guidance for forecasters tasked with their issuance.

Figure 2 presents an HREFv2 plot of the NMEP for 2-5 km (AGL) UH $>75 \mathrm{~m}^{2} \mathrm{~s}^{-2}$ over the 24 -h convective day for 19 July 2018 (this case will also be used for subsequent plots). As described previously, $\mathrm{UH}$ is a diagnostic parameter intended to identify rotating

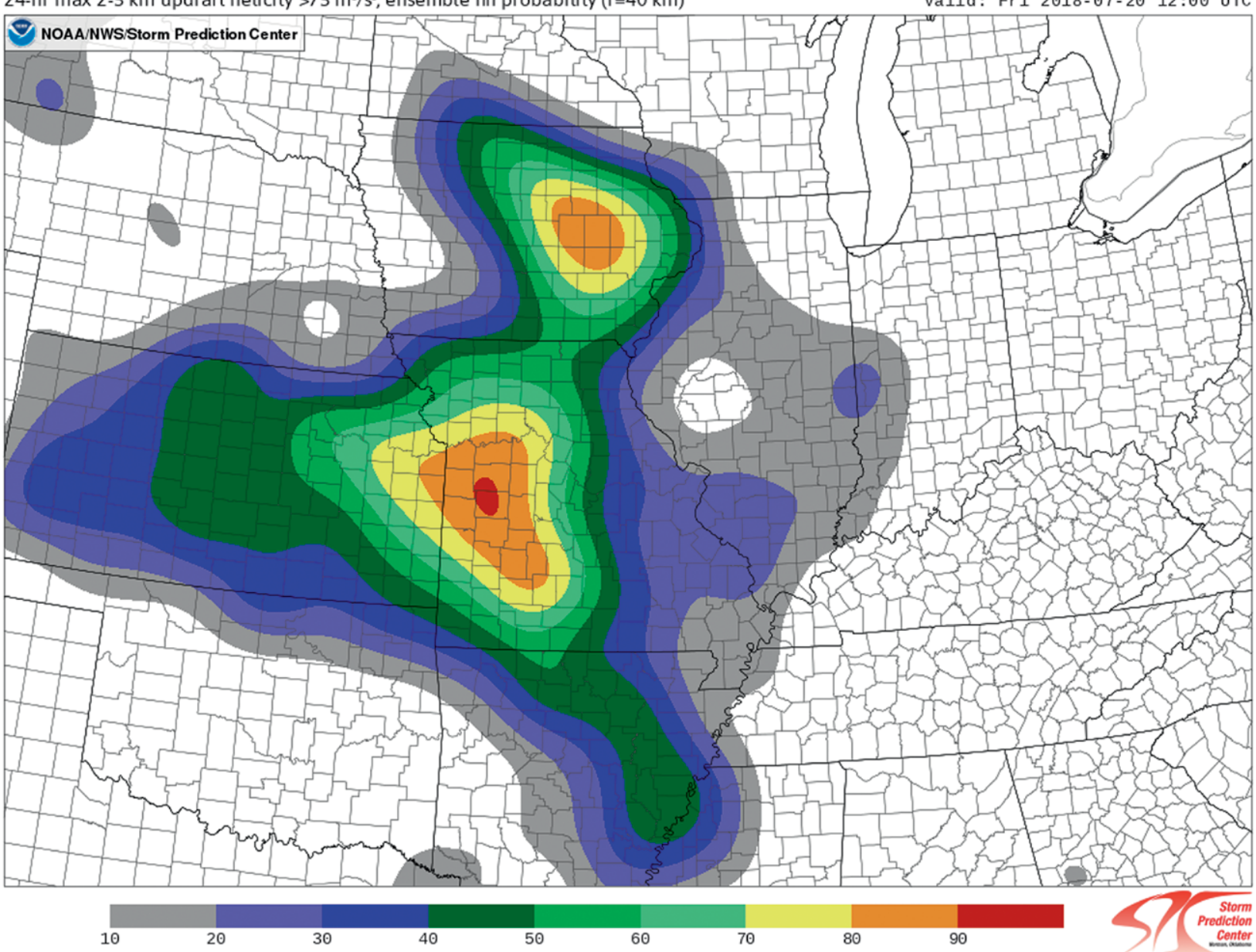

Fig. 2. From the 1200 UTC 19 Jul 2018 cycle of the HREFv2, NMEP for 2-5 km UH >75 m $\mathrm{s}^{-2}$ over the 24-h period from I 200 UTC 19 Jul to 1200 UTC 20 Jul 2018 is plotted as a color fill. The NMEP field has been computed and smoothed in the manner described in the main text, and is expressed as a percentage. 
thunderstorm updrafts in simulated convective storms. ${ }^{5}$ The NMEP field has two local maxima of note: one in east-central Iowa, and another in western Missouri. Despite the smoothing applied in the final step of postprocessing, calculating the NMEP on the 3-km HREFv2 grid has afforded sufficient spatial resolution to distinguish variability in threat probability across different regions of a state with appropriate levels of detail that are consistent with the uncertainty involved in forecasting convective storms. While an NMEP plot of this type is an ideal high-level summary of the probabilistic ensemble forecast for rotating updrafts, important information about the individual member $\mathrm{UH}$ forecasts and ensemble distribution of $\mathrm{UH}$ magnitude cannot be discerned on this basis alone. In the next section, other approaches for HMF visualization are described that elucidate member forecasts explicitly.

\section{VISUALIZATION OF ENSEMBLE MEMBER}

HMFS. To complement the aggregation of member data during postprocessing to compute a single ensemble summary field (e.g., ensemble mean or NMEP), an alternative ensemble visualization approach is to present individual member data directly to the user. The so-called "spaghetti" diagram (e.g., Toth et al. 1997) is a prototypical visualization of this type that historically has found use in operational settings, and is particularly well suited to presenting the ensemble distribution of key synoptic features (e.g., member contours for a single value of $500-\mathrm{mb}$ geopotential height). When CAMs are used for forecasting convection and other phenomena on smaller spatiotemporal scales, traditional approaches in this class of visualizations can become extremely noisy, and therefore less practical. Some alternative approaches currently employed for CAM output in the context of convective-scale forecasting are now described.

Paintball plot. The paintball plot is a close cousin of the spaghetti diagram, but modified to optimize for highlighting numerous discrete features in a discontinuous field (e.g., a relatively large simulated radar reflectivity threshold associated with intense thunderstorm cores), rather than smooth features in a continuous field (e.g., a climatologically normal $500-\mathrm{mb}$ geopotential height threshold). The primary distinction is that on a paintball plot, color fill is used wherever a threshold is exceeded on the grid, whereas only the threshold contour is drawn on a traditional spaghetti diagram. Additionally, on a paintball plot, each ensemble member is assigned a distinct color. ${ }^{6}$ In some cases, partial transparency of the color fill may be employed, making it easier for the user to discern the location and extent of feature overlap between members.

Figure 3 presents a paintball plot for the same HREFv2 UH forecast depicted in Fig. 2. The paintball threshold is $75 \mathrm{~m}^{2} \mathrm{~s}^{-2}$, and NMEP contours for that threshold are overlaid. Compared with Fig. 2, important new details emerge. First, the path lengths and orientations of member UH swaths are easily seen. It is clear that rotating updraft tracks in central Iowa are relatively short and primarily moving east or east-northeastward, whereas those in western Missouri are relatively long and primarily moving south-southeastward. ${ }^{7}$ Second, the swaths in both areas are relatively narrow in width, and swaths common to a single ensemble member are spatially distinct from one another. This implies a tendency for discrete supercellular structures in the CAM forecasts, rather than a mesoscale convective system. Third, the color scheme employed uses lighter colors for the time-lagged members and darker colors for the nonlagged members to identify forecast trends between the previous and current cycle of deterministic CAMs (e.g., latest runs trending away from UH swaths in north-central Iowa in Fig. 3). Finally, the color scheme employed also uses warm colors for WRF-ARW members and cool colors for NMMB members for identification of model solutions clustering by dynamic core (e.g., UH swaths in Iowa in Fig. 3 from WRF-ARW members are farther east toward east-central Iowa than the NMMB members).

\footnotetext{
${ }^{5}$ The magnitude of UH best corresponding to a threat for particular severe weather hazards (e.g., tornadoes) will vary across modeling systems based on grid spacing, model core, physics parameterizations, and other relevant attributes. For the SPC HREF Ensemble Viewer, we display thresholds of 75 and $150 \mathrm{~m}^{2} \mathrm{~s}^{-2}$ based on preliminary tests and forecaster impressions. In the future, the availability of model UH climatologies for each HREF member calculated over a sufficiently large sample of events may support a refinement of these thresholds.

${ }^{6}$ Spaghetti diagrams may also use distinct colors for each member, although this varies in practice.

${ }^{7}$ In principle, the storm motion direction of features associated with HMF swaths could be either of two diametrically opposed possibilities; in practice, forecasters will typically know which of these two directions is plausible (although this may not be true in cases where the expected storm motion magnitude is very small).
} 
In principle, any criterion or combination of criteria could be leveraged in the mapping of ensemble members to a paintball color scheme, depending on the dimension(s) of variability within the membership [e.g., model cores, time-lag offsets, parameterization schemes, initial conditions (ICs), IC perturbations]. A CAM super-ensemble like the Community Leveraged Unified Ensemble (CLUE; Clark et al. 2018) is also suitable for paintball plots in which each ensemble subset is assigned a single color, or even a base color from which each of the subset's members deviates slightly.

Ensemble maximum HMFs. Another approach for displaying the full set of member HMFs is to plot the ensemble maximum over a relevant time bin. While ensemble maximum fields are also a common display type for traditional NWP fields, the interpretation of ensemble maximum HMFs in CAMs can differ subjectively. In particular, because of the small spatial scales of HMF extrema, discernible swaths representing intense convection in individual members typically dominate these plots. The primary advantage an ensemble maximum HMF plot holds over a paintball HMF plot is that the full ensemble distribution of HMF magnitude can be discerned [e.g., do the most intense simulated storms exhibit very strong updraft rotation (i.e., $\mathrm{UH} \geq 150 \mathrm{~m}^{2} \mathrm{~s}^{-2}$ at $3-\mathrm{km}$ grid spacing) suggestive of more intense supercell potential, or only weak-to-moderate rotation?]. Conversely, a primary disadvantage is that this approach does not differentiate between individual member solutions, such that four swaths of large UH values in the ensemble maximum could represent either 1) a single member with four supercells; 2) four members producing a single supercell each, and each member's storm is spatially displaced from the other members' storms; or 3) some combination of the two possibilities. Thus,

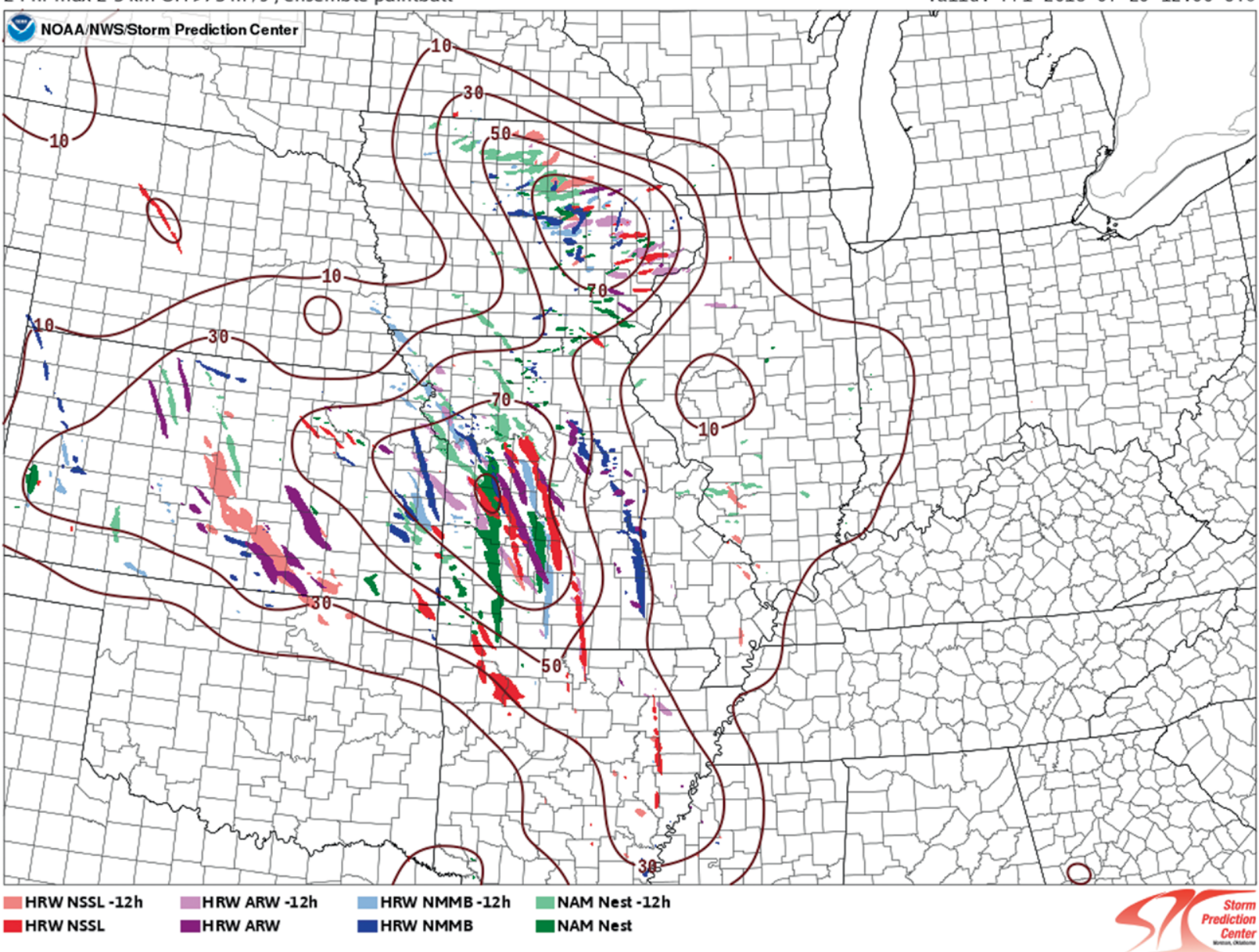

Fig. 3. From the 1200 UTC 19 Jul 2018 cycle of the HREFv2, the paintball plot for $2-5 \mathrm{~km} \mathrm{UH}>75 \mathrm{~m}^{2} \mathrm{~s}^{-2}$ over the 24-h period from I200 UTC 19 Jul to 1200 UTC 20 Jul 2018. Contours of NMEP for 2-5 km UH >75 m s s-2 (the same field plotted as color fill in Fig. 2) are overlaid. 
it is advisable to overlay contours of NMEP on an ensemble maximum HMF plot to provide probabilistic context. For an astute user, this combination of ensemble maximum and NMEP fields provides much of the useful ensemble information regarding the HMF in a single plot.

Figure 4 presents an ensemble maximum plot for the same UH forecast shown in Fig. 3, with the same $\mathrm{UH}>75 \mathrm{~m}^{2} \mathrm{~s}^{-2}$ NMEP contours overlaid. The path length, path width, and orientation of member $\mathrm{UH}$ swaths are again broadly discernable, albeit with less evident member-to-member distinction than in the equivalent paintball plot. However, information about the UH magnitude distribution that was absent in Figs. 2 and 3 is now readily seen, and these data can have important implications on the magnitude and conditionality of severe weather threats in different areas. Despite modest NMEP values, relatively large magnitudes of $\mathrm{UH}\left(>300 \mathrm{~m}^{2} \mathrm{~s}^{-2}\right)$ are present in the ensemble maximum field over southwest Kansas, suggesting a very conditional threat for an intense supercell thunderstorm (a low-probability, high-impact event). By comparison, ensemble maximum UH values over western Missouri are similar in magnitude to those over southwest Kansas, but the NMEP contours suggest far greater likelihood of intense supercell occurrence (a high-probability, high-impact event).

Postage stamps and member viewer. It can also be beneficial to plot standalone ensemble member fields in the same framework used for deterministic models. The so-called "postage stamp" plot is a traditional example constructed by creating small subplots of a field for each member in a grid-like configuration, allowing all members to be viewed side by side with the benefit of comparative context. A potential challenge for leveraging postage stamp plots in CAM

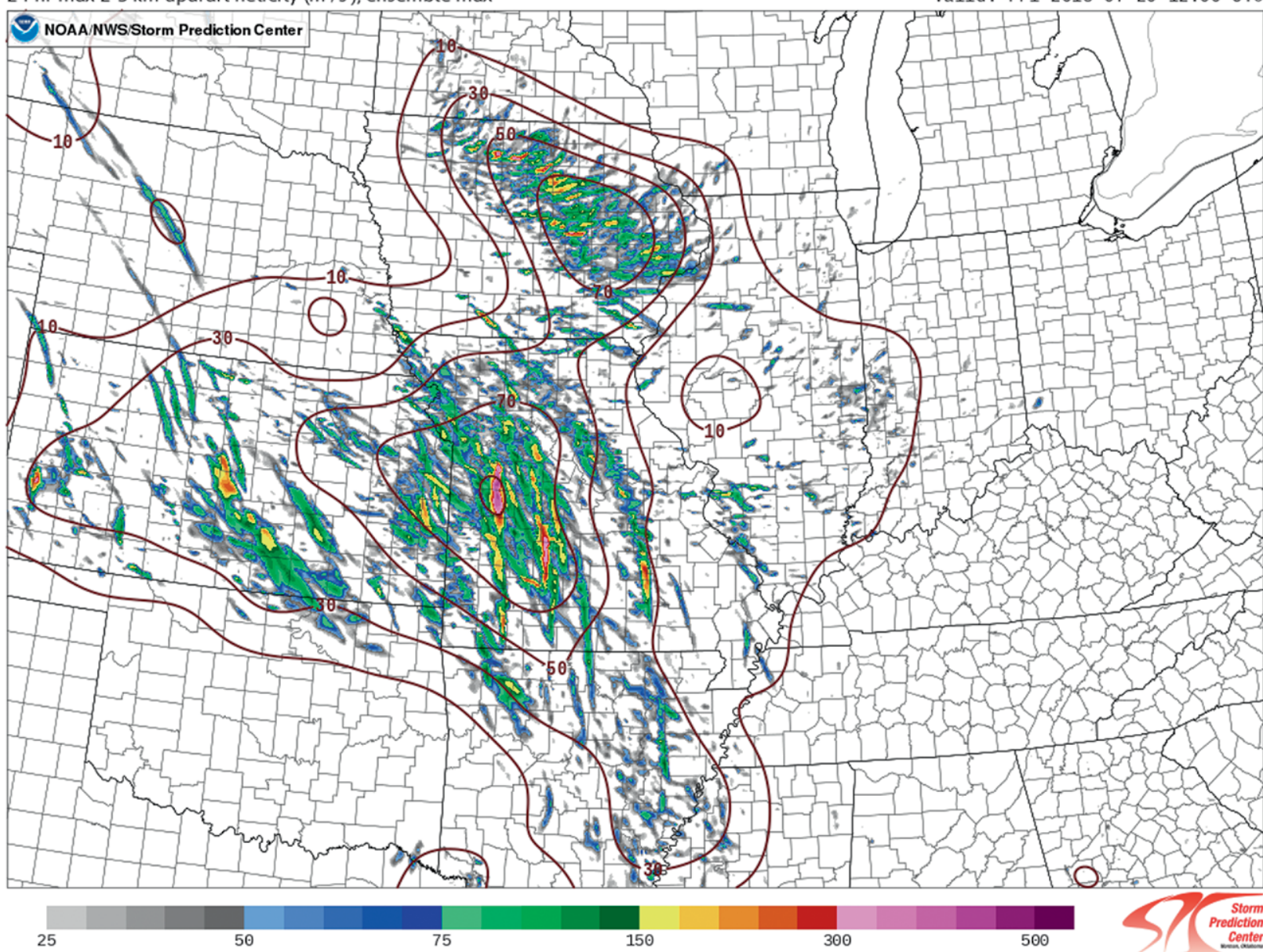

Fig. 4. As in Fig. 3, but the color fill field is the ensemble maximum of the 24-h maximum 2-5 $\mathrm{km}$ UH for the period. 
ensemble displays is the relatively small spatial scale of important features in HMFs and similar fields. In many cases, this may limit the practicality of HMF postage stamp plots to geographically smaller domains (e.g., a mesoscale region, single state or smaller). An example postage stamp plot of composite reflectivity for the HREFv2 is presented in Fig. 5.

An alternate approach is using a "member viewer," wherein full-size plots are created for each member, and the end user has the ability to toggle through member forecasts in a seamless and sequential manner. The member viewer must be implemented within the display framework [e.g., an Advanced Weather Interactive Processing System (AWIPS) workstation or a website], rather than within the plot itself. The SPC HREF Ensemble Viewer generally follows the approach first introduced on the NCAR Real Time Ensemble Forecast website, which displayed daily forecasts from the experimental NCAR Ensemble Prediction System (Schwartz et al. 2015) when it was run in real time from 2015 to 2017. In this browserbased implementation, the user hovers over or taps a member's label to activate the corresponding plot in the main display area. Figure 6 presents an example of the member viewer on the SPC HREF Ensemble Viewer for reflectivity, with regions of HMF UH $>75 \mathrm{~m}^{2} \mathrm{~s}^{-2}$ filled in translucent black to highlight simulated supercell tracks.

As forecasters familiar with deterministic CAMs begin adding CAM ensembles to their toolbox, postage stamp and member plots can potentially soften the learning curve and facilitate trust in the value of ensemble summary fields. Given the relationship between radar observed storm structures and severe weather hazards, SPC forecasters have found it prudent to assess individual member

\section{HREF}

Composite reflectivity (dBZ), ensemble postage stamp
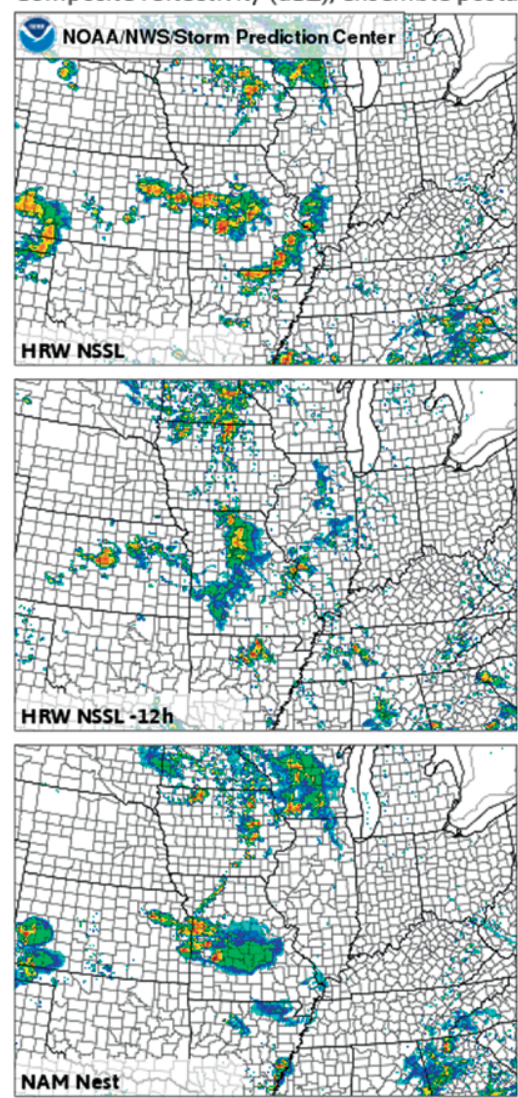
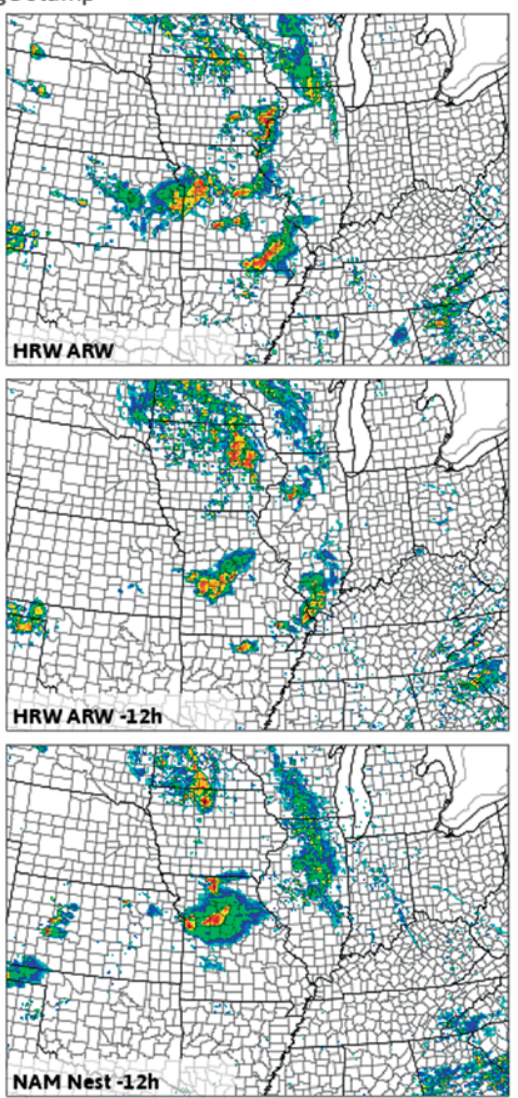

Run: Thu 2018-07-19 12:00 UTC Valid: Thu 2018-07-19 22:00 UTC

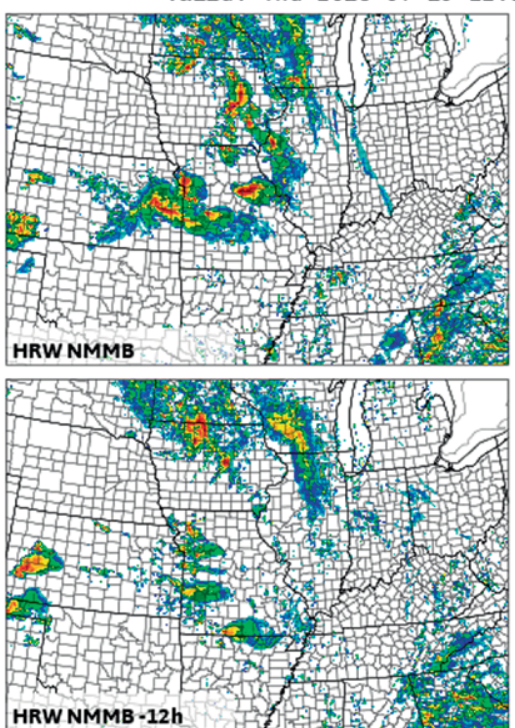

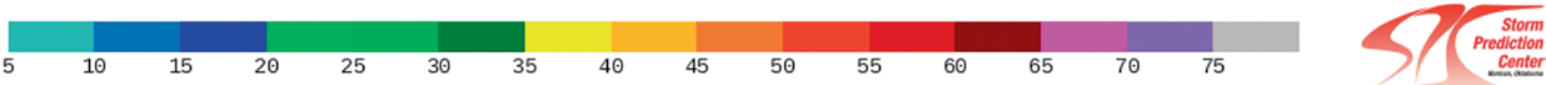

FIG. 5. Postage stamp plot from the 1200 UTC 19 Jul 2018 HREFv2 cycle. Plotted data are a 10 -h forecast valid at 22 UTC 19 Jul 2018 and depict simulated composite reflectivity as color fill. Data for each HREFv2 member appear in a subplot; the grid of subplots is arranged such that, for any CAM configuration (e.g., HRW NSSL), the latest and time-lagged members from that configuration are adjacent to one another. 
forecasts, especially of simulated reflectivity, to provide predicted storm-scale context for the ensemble statistical properties. Most forecasters at the SPC, for example, prefer to conceptualize a convective scenario (or multiple potential scenarios) during the formulation of forecast products. As such, they are unlikely to utilize an NMEP field in isolation without interrogating the underlying deterministic solutions of each member, verifying that most or all of these solutions are plausible within the forecaster's existing conceptual model derived from observational data, experience, and traditional NWP guidance.

\section{CONCLUDING REMARKS AND THE FUTURE OF CAM ENSEMBLE DISPLAYS.}

The operational implementation of the HREFv2 at NCEP in 2017 was a landmark achievement for the NWP enterprise in the United States, ushering in a new era in which CAM ensembles will be increasingly regarded as essential tools for a variety of hazardous weather forecasting applications, including severe convection, heavy rain and flooding, winter weather, and fire weather. Although many
NWP users have become familiar with deterministic CAMs over the past decade, interpreting the output from a CAM ensemble system requires understanding some new paradigms. In this article, we have summarized several key postprocessing and display approaches currently employed for CAM ensembles in frameworks like the SPC HREF Ensemble Viewer (www.spc.noaa.gov/exper/href) in the context of convective forecasting. Of particular importance is the ensemble treatment of storm-attribute HMFs like $\mathrm{UH}$, updraft speed, and near-ground wind speed. In these fields, key features are associated with explicitly predicted convective structures and have inherently small spatiotemporal scales, requiring fundamentally different ensemble processing, display formats, and forecaster interpretation than traditional NWP fields. Examples of ensemble display approaches currently employed for HMFs include spatial neighborhoodbased computations like the NMEP; paintball plots, which are closely related to spaghetti diagrams; ensemble maximum fields with an NMEP field overlaid; and displays of member solutions, such as postage stamp plots or the web-based member viewer.
HREF Member. HRW NSSL

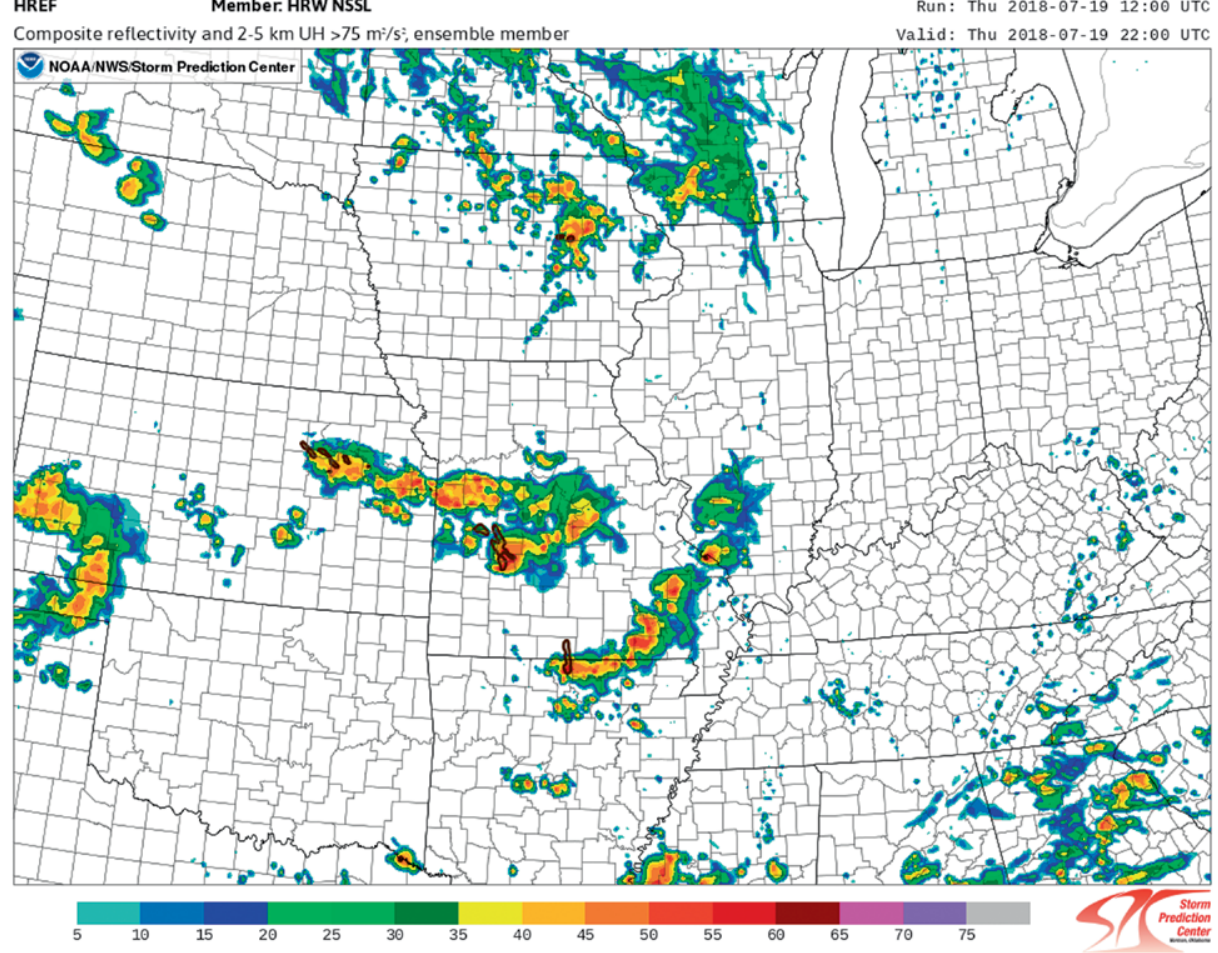

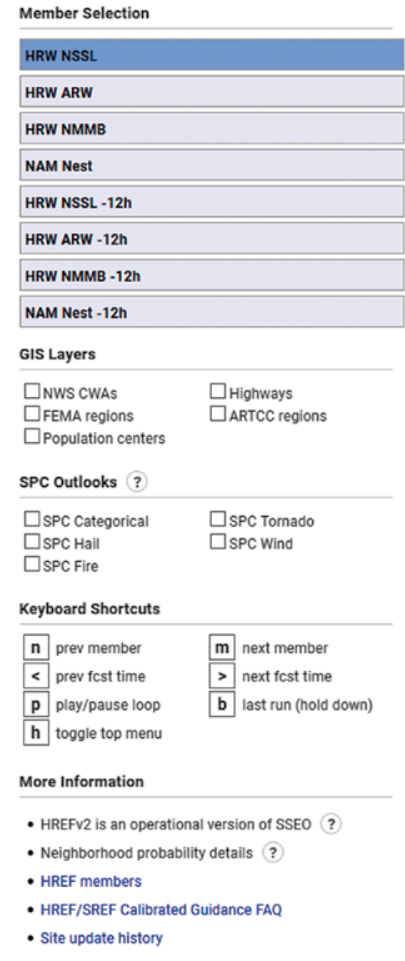

FIG. 6. Member viewer on the SPC HREF Ensemble Viewer displaying data from the 1200 UTC 19 Jul 2018 HREFv2 cycle. Plotted data are a 10-h forecast valid at 2200 UTC 19 Jul 2018 and depict simulated composite reflectivity as color fill, with outlined translucent black regions denoting I-h maximum 2-5 km $\mathrm{UH}^{>} 75 \mathrm{~m}^{2} \mathrm{~s}^{-2}$ over the previous hour. The HRW NSSL member is selected for display here, as indicated by the darker blue shading of its label on the menu at right; hovering over another member in the list (or using a keyboard shortcut) immediately switches the display to that member. 
Each of these display types communicates a different slice of information about the ensemble distribution of an HMF. Using multiple display types in a complementary manner is likely to provide a forecaster with a more thorough understanding of the probability and magnitude of potential weather hazards.

Real-time CAM ensemble systems are still in their early development, and new diagnostics, postprocessing approaches, and visualizations are active areas of research and testing. Moving forward, the Warn-onForecast (WoF) paradigm calls for the real-time use of larger CAM ensembles at higher resolution and shorter time scales relative to the HREFv2 (Stensrud et al. 2009). As these more advanced ensembles are developed, and as subhourly temporal information becomes increasingly critical to maximizing their operational utility in the spirit of WoF, new avenues for displaying and interpreting these data will emerge. The NSSL Experimental WoF System for Ensembles (NEWS-e; Wheatley et al. 2015; Jones et al. 2016), a prototype WoF system run experimentally at the National Severe Storms Laboratory, has provided a testing ground for early exploration in this area. One potential approach pioneered with the NEWS-e is the display of ensemble percentile values for HMFs. In studying the responses of meteorologists surveyed on their interpretations of probabilistic and percentilebased products from the NEWS-e, Wilson et al. (2019) found a compelling need for improved knowledge and understanding of this type ensemble information in the operational and research communities. This serves as a stark reminder that effective training must supplement the proliferation of new postprocessing techniques in this arena. Object-based methods for identifying some features of interest in HMFs have also been explored in recent research endeavors involving CAMs (e.g., Clark et al. 2012b; Sobash et al. 2016a; Skinner et al. 2018), and these may prove increasingly relevant to real-time CAM postprocessing with further refinement. In the longer term, as NWP evolves toward global CAM ensembles and much smaller horizontal grid spacing (e.g., sufficient to resolve important details and substructures within an individual convective storm), robust parallel efforts to adapt postprocessing and visualization approaches will be essential to leveraging these rich datasets.

ACKNOWLEDGMENTS. The authors thank all previous participants and facilitators in the annual NOAA Hazardous Weather Testbed Spring Forecasting Experiment, where many seeds were planted over the years for the visualizations and postprocessing techniques described in this article. We also thank Matthew Pyle and others at the NOAA Environmental Modeling Center and NCEP Central Operations for their work in configuring and implementing the HREFv2. We are grateful to Trevor Alcott and another anonymous reviewer for their thorough and insightful comments, which have helped to improve this manuscript considerably.

The first author was provided support by NOAA/ Office of Oceanic and Atmospheric Research under NOAA-University of Oklahoma Cooperative Agreement NA16OAR4320115, U.S. Department of Commerce. Authors AJC and JSK completed this work as part of regular duties at the federally funded NOAA National Severe Storms Laboratory. Authors ILJ and SJW completed this work as part of regular duties at the federally funded NOAA Storm Prediction Center.

\section{REFERENCES}

Ahijevych, D., E. Gilleland, B. G. Brown, and E. E. Ebert, 2009: Application of spatial verification methods to idealized and NWP-gridded precipitation forecasts. Wea. Forecasting, 24, 1485-1497, https://doi .org/10.1175/2009WAF2222298.1.

Aligo, E. A., B. Ferrier, and J. R. Carley, 2018: Modified NAM microphysics for forecasts of deep convective storms. Mon. Wea. Rev., 146, 4115-4153, https://doi .org/10.1175/MWR-D-17-0277.1.

Blake, B. T., J. R. Carley, T. I. Alcott, I. Jankov, M. E. Pyle, S. E. Perfater, and B. Albright, 2018: An adaptive approach for the calculation of ensemble gridpoint probabilities. Wea. Forecasting, 33, 1063-1080, https://doi.org/10.1175/WAF-D-18-0035.1.

Clark, A. J., W. A. Gallus, and T.-C. Chen, 2007: Comparison of the diurnal precipitation cycle in convection-resolving and non-convection-resolving mesoscale models. Mon. Wea. Rev., 135, 3456-3473, https://doi.org/10.1175/MWR3467.1.

- and Coauthors, 2012a: An overview of the 2010 Hazardous Weather Testbed Experimental Forecast Program spring experiment. Bull. Amer. Meteor. Soc., 93, 55-74, https://doi.org/10.1175 /BAMS-D-11-00040.1.

—, J. S. Kain, P. T. Marsh, J. Correia, M. Xue, and F. Kong, 2012b: Forecasting tornado pathlengths using a three-dimensional object identification algorithm applied to convection-allowing forecasts. Wea. Forecasting, 27, 1090-1113, https://doi.org/10.1175 /WAF-D-11-00147.1.

- , and Coauthors, 2018: The Community Leveraged Unified Ensemble (CLUE) in the 2016 NOAA/ Hazardous Weather Testbed Spring Forecasting Experiment. Bull. Amer. Meteor. Soc., 99, 1433-1448, https://doi.org/10.1175/BAMS-D-16-0309.1. 
Dey, S. R. A., R. S. Plant, N. M. Roberts, and S. Migliorini, 2016: Assessing spatial precipitation uncertainties in a convective-scale ensemble. Quart. J. Roy. Meteor. Soc., 142, 2935-2948, https://doi.org/10.1002/qj .2893 .

Done, J., C. A. Davis, and M. Weisman, 2004: The next generation of NWP: Explicit forecasts of convection using the Weather Research and Forecasting (WRF) model. Atmos. Sci. Lett., 5, 110-117, https:// doi.org/10.1002/asl.72.

Droegemeier, K. K., 1997: The numerical prediction of thunderstorms: Challenges, potential benefits and results from real-time operational tests. WMO Bull., 46, 324-336.

Ebert, E. E., 2009: Neighborhood verification: A strategy for rewarding close forecasts. Wea. Forecasting, 24, 1498-1510, https://doi.org/10.1175 /2009WAF2222251.1.

Ferrier, B., 1994: A double-moment multiple-phase four-class bulk ice scheme. Part I: Description. J. Atmos. Sci., 51, 249-280, https://doi.org/10.1175/1520 -0469(1994)051<0249:ADMMPF>2.0.CO;2.

Gallo, B. T., A. J. Clark, and S. R. Dembek, 2016: Forecasting tornadoes using convection-permitting ensembles. Wea. Forecasting, 31, 273-295, https:// doi.org/10.1175/WAF-D-15-0134.1.

— , and Coauthors, 2017: Breaking new ground in severe weather prediction: The 2015 NOAA/ Hazardous Weather Testbed Spring Forecasting Experiment. Wea. Forecasting, 32, 1541-1568, https:// doi.org/10.1175/WAF-D-16-0178.1.

Guyer, J. L., and I. L. Jirak, 2014: The utility of stormscale ensemble forecasts of cool season severe weather events from the SPC perspective. 27th Conf. on Severe Local Storms, Madison, WI, Amer. Meteor. Soc., P3.37, https://ams.confex.com/ams/27SLS /webprogram/Paper254640.html.

Hong, S.-Y., and J.-O. J. Lim, 2006: The WRF singlemoment 6-class microphysics scheme (WSM6). J. Korean Meteor. Soc, 42, 129-151.

_-, Y. Noh, and J. Dudhia, 2006: A new vertical diffusion package with an explicit treatment of entrainment processes. Mon. Wea. Rev., 134, 2318-2341, https://doi.org/10.1175/MWR3199.1.

Janjić, Z. I., 1994: The step-mountain eta coordinate model: Further developments of the convection, viscous sublayer, and turbulence closure schemes. Mon. Wea. Rev., 122, 927-945, https://doi .org/10.1175/1520-0493(1994)122<0927:TSMECM $>2.0 . \mathrm{CO} ; 2$.

— , and R. L. Gall, 2012: Scientific documentation of the NCEP nonhydrostatic multiscale model on the $B$ grid (NMMB). Part 1: Dynamics. NCAR Tech. Note
NCAR/TN-489+STR, 75 pp., https://doi.org/10.5065 /D6WH2MZX.

Jirak, I. L., S. J. Weiss, and C. J. Melick, 2012: The SPC storm-scale ensemble of opportunity: Overview and results from the 2012 Hazardous Weather Testbed Spring Forecasting Experiment. 26th Conf. on Severe Local Storms, Nashville, TN, Amer. Meteor. Soc., P9.137, https://ams.confex.com/ams/26SLS /webprogram/Paper211729.html.

_ C. J. Melick, and S. J. Weiss, 2014: Combining probabilistic ensemble information from the environment with simulated storm attributes to generate calibrated probabilities of severe weather hazards. 27th Conf. on Severe Local Storms, Madison, WI, Amer. Meteor. Soc., P2.5, https://ams.confex.com /ams/27SLS/webprogram/Paper254649.html.

— , A. J. Clark, B. Roberts, B. T. Gallo, and S. J. Weiss, 2018: Exploring the optimal configuration of the High Resolution Ensemble Forecast System. 25th Conf. on Numerical Weather Prediction, Denver, CO, Amer. Meteor. Soc., 14B.6, https://ams.confex.com /ams/29WAF25NWP/webprogram/Paper345640 .html.

Jones, T. A., K. Knopfmeier, D. Wheatley, G. Creager, P. Minnis, and R. Palikonda, 2016: Storm-scale data assimilation and ensemble forecasting with the NSSL Experimental Warn-on-Forecast System. Part II: Combined radar and satellite data experiments. Wea. Forecasting, 31, 297-327, https://doi.org/10.1175 /WAF-D-15-0107.1.

Jung, Y., G. Zhang, and M. Xue, 2008: Assimilation of simulated polarimetric radar data for a convective storm using the ensemble Kalman filter. Part I: Observation operators for reflectivity and polarimetric variables. Mon. Wea. Rev., 136, 2228-2245, https://doi.org/10.1175/2007MWR2083.1.

Kain, J. S., S. J. Weiss, J. J. Levit, M. E. Baldwin, and D. R. Bright, 2006: Examination of convection-allowing configurations of the WRF Model for the prediction of severe convective weather: The SPC/NSSL Spring Program 2004. Wea. Forecasting, 21, 167-181, https:// doi.org/10.1175/WAF906.1.

— , and Coauthors, 2008: Some practical considerations regarding horizontal resolution in the first generation of operational convection-allowing NWP. Wea. Forecasting, 23, 931-952, https://doi .org/10.1175/WAF2007106.1.

— , S. R. Dembek, S. J. Weiss, J. L. Case, J. J. Levit, and R. A. Sobash, 2010: Extracting unique information from high-resolution forecast models: Monitoring selected fields and phenomena every time step. Wea. Forecasting, 25, 1536-1542, https://doi .org/10.1175/2010WAF2222430.1. 
Koch, S. E., B. Ferrier, M. T. Stoelinga, E. Szoke, S. J. Weiss, and J. S. Kain, 2005: The use of simulated radar reflectivity fields in the diagnosis of mesoscale phenomena from high-resolution WRF model forecasts. 11th Conf. on Mesoscale Processes, Albuquerque, NM, Amer. Meteor. Soc., J4J.7, https:// ams.confex.com/ams/pdfpapers/97032.pdf.

Krocak, M., and H. E. Brooks, 2017: Towards consistency in forecasting severe weather events across a wide range of temporal and spatial scales in the FACETS paradigm. Seventh Conf. on Transition of Research to Operations, Seattle, WA, Amer. Meteor. Soc., J4.6, https://ams.confex.com/ams/97Annual /webprogram/Paper308117.html.

Lilly, D. K., 1990: Numerical prediction of thunderstorms-Has its time come? Quart. J. Roy. Meteor. Soc., 116, 779-798, https://doi.org/10.1002/qj .49711649402 .

Liu, C., M. W. Moncrieff, J. D. Tuttle, and R. E. Carbone, 2006: Explicit and parameterized episodes of warm-season precipitation over the continental United States. Adv. Atmos. Sci., 23, 91-105, https://doi .org/10.1007/s00376-006-0010-9.

Schwartz, C. S., and R. A. Sobash, 2017: Generating probabilistic forecasts from convection-allowing ensembles using neighborhood approaches: A review and recommendations. Mon. Wea. Rev., 145, 33973418, https://doi.org/10.1175/MWR-D-16-0400.1.

— G. S. Romine, R. A. Sobash, K. R. Fossell, and M. L. Weisman, 2015: NCAR's experimental real-time convection-allowing ensemble prediction system. Wea. Forecasting, 30, 1645-1654, https://doi.org/10.1175 /WAF-D-15-0103.1.

Skamarock, W. C., and Coauthors, 2008: A description of the Advanced Research WRF version 3. NCAR Tech. Note NCAR/TN-475+STR, 113 pp., https:// doi.org/10.5065/D68S4MVH.

Skinner, P. S., and Coauthors, 2018: Object-based verification of a prototype Warn-on-Forecast system. Wea. Forecasting, 33, 1225-1250, https://doi.org/10.1175 /WAF-D-18-0020.1.

Sobash, R. A., J. S. Kain, D. R. Bright, A. R. Dean, M. C. Coniglio, and S. J. Weiss, 2011: Probabilistic forecast guidance for severe thunderstorms based on the identification of extreme phenomena in convectionallowing model forecasts. Wea. Forecasting, 26, 714-728, https://doi.org/10.1175/WAF-D-10-05046.1.

- , G. S. Romine, C. S. Schwartz, D. J. Gagne, and M. L. Weisman, 2016a: Explicit forecasts of low-level rotation from convection-allowing models for nextday tornado prediction. Wea. Forecasting, 31, 15911614, https://doi.org/10.1175/WAF-D-16-0073.1.

, C. S. Schwartz, G. S. Romine, K. R. Fossell, and M. L. Weisman, 2016b: Severe weather prediction using storm surrogates from an ensemble forecasting system. Wea. Forecasting, 31, 255-271, https://doi .org/10.1175/WAF-D-15-0138.1.

Stensrud, D. J., and Coauthors, 2009: Convectivescale Warn-on-Forecast System: A vision for 2020. Bull. Amer. Meteor. Soc., 90, 1487-1499, https://doi .org/10.1175/2009BAMS2795.1.

Toth, Z., E. Kalnay, S. M. Tracton, R. Wobus, and J. Irwin, 1997: A synoptic evaluation of the NCEP ensemble. Wea. Forecasting, 12, 140-153, https:// doi.org/10.1175/1520-0434(1997)012<0140:ASEOTN $>2.0 . \mathrm{CO} ; 2$.

Weisman, M. L., W. C. Skamarock, and J. B. Klemp, 1997: The resolution dependence of explicitly modeled convective systems. Mon. Wea. Rev., 125, 527-548, https://doi.org/10.1175/1520-0493(1997)125<0527: TRDOEM $>2.0 . \mathrm{CO} ; 2$.

- C. Davis, W. Wang, K. W. Manning, and J. B. Klemp, 2008: Experiences with 0-36-h explicit convective forecasts with the WRF-ARW model. Wea. Forecasting, 23, 407-437, https://doi .org/10.1175/2007WAF2007005.1.

Wheatley, D. M., K. H. Knopfmeier, T. A. Jones, and G. J. Creager, 2015: Storm-scale data assimilation and ensemble forecasting with the NSSL Experimental Warn-on-Forecast System. Part I: Radar data experiments. Wea. Forecasting, 30, 1795-1817, https://doi .org/10.1175/WAF-D-15-0043.1.

Wilson, K. A., P. L. Heinselman, P. S. Skinner, J. J. Choate, and K. E. Klockow-McClain, 2019: Meteorologists' interpretations of storm-scale ensemble-based forecast guidance. Wea. Climate Soc., 11, 337-354, https://doi.org/10.1175/WCAS-D-18-0084.1.

Xue, M., D. Wang, J. Gao, K. Brewster, and K. K. Droegemeier, 2003: The Advanced Regional Prediction System (ARPS), storm-scale numerical weather prediction and data assimilation. Meteor. Atmos. Phys., 82, 139-170.

— , and Coauthors, 2007: CAPS real-time storm-scale ensemble and high-resolution forecasts as part of the NOAA Hazardous Weather Testbed 2007 spring experiment. 18th Conf. on Numerical Weather Prediction, Park City, UT, Amer. Meteor. Soc., 3B.1, https:// ams.confex.com/ams/pdfpapers/124587.pdf. 